Impact of minority concentration on fundamental $(H) D$ ICRF heating performance in JET-ILW

This content has been downloaded from IOPscience. Please scroll down to see the full text.

2014 Nucl. Fusion 54073006

(http://iopscience.iop.org/0029-5515/54/7/073006)

View the table of contents for this issue, or go to the journal homepage for more

Download details:

IP Address: 193.190.205.100

This content was downloaded on 04/11/2014 at 13:33

Please note that terms and conditions apply. 


\title{
Impact of minority concentration on fundamental (H)D ICRF heating performance in JET-ILW
}

\author{
E. Lerche ${ }^{1,2}$, D. Van Eester ${ }^{1}$, P. Jacquet ${ }^{2}$, M.-L. Mayoral ${ }^{2}$, \\ V. Bobkov ${ }^{3}$, L. Colas ${ }^{4}$, A. Czarnecka ${ }^{5}$, K. Crombé ${ }^{1}$, I. Monakhov ${ }^{2}$, \\ F. Rimini ${ }^{2}$, M. Santala ${ }^{6}$ and JET-EFDA Contributors ${ }^{\mathrm{a}}$ \\ JET-EFDA, Culham Science Centre, Abingdon, OX14 3DB, UK \\ ${ }^{1}$ Association EURATOM-Belgian State, LPP-ERM-KMS, TEC partner, Brussels, Belgium \\ ${ }^{2}$ Euratom/CCFE Fusion Association, Culham Science Centre, Abingdon, OX14 3DB, UK \\ ${ }^{3}$ Max-Planck-Institut für Plasmaphysik, EURATOM-Assoziation, Garching, Germany \\ ${ }^{4}$ CEA, IRFM, F-13108 Saint-Paul-Lez-Durance, France \\ ${ }^{5}$ Association Euratom-IPPLM, Hery 23, 01-497 Warsaw, Poland \\ ${ }^{6}$ Helsinki University of Technology, Tekes-Euratom Association, Finland \\ E-mail: ernesto.lerche@jet.efda.org
}

Received 2 February 2014, revised 14 March 2014

Accepted for publication 24 March 2014

Published 22 April 2014

\begin{abstract}
ITER will start its operation with non-activated hydrogen and helium plasmas at a reduced magnetic field of $B_{0}=2.65 \mathrm{~T}$. In hydrogen plasmas, the two ion cyclotron resonance frequency (ICRF) heating schemes available for central plasma heating (fundamental $\mathrm{H}$ majority and $2 \mathrm{nd}$ harmonic ${ }^{3} \mathrm{He}$ minority ICRF heating) are likely to suffer from relatively low RF wave absorption, as suggested by numerical modelling and confirmed by previous JET experiments conducted in conditions similar to those expected in ITER's initial phase. With ${ }^{4} \mathrm{He}$ plasmas, the commonly adopted fundamental $\mathrm{H}$ minority heating scheme will be used and its performance is expected to be much better. However, one important question that remains to be answered is whether increased levels of hydrogen (due to e.g. H pellet injection) jeopardize the high performance usually observed with this heating scheme, in particular in a full-metal environment. Recent JET experiments performed with the ITER-like wall shed some light onto this question and the main results concerning ICRF heating performance in L-mode discharges are summarized here.
\end{abstract}

Keywords: ICRF heating, RF waves, tokamak

(Some figures may appear in colour only in the online journal)

\section{Introduction}

For minimizing neutron induced activation, ITER will first operate using hydrogen $(\mathrm{H})$ and helium $\left({ }^{4} \mathrm{He}\right)$ plasmas at half the nominal magnetic field, $B_{0}=2.65 \mathrm{~T}$. With $16.5 \mathrm{MW}$ of neutral-beam injection (NBI) and $15 \mathrm{MW}$ of electron cyclotron resonance heating $(\mathrm{ECRH})$ power expected to be available in the initial operation phase, simulations have shown that L-mode plasmas with central electron densities of $n_{\mathrm{e}}=3.5 \times$ $10^{19} \mathrm{~m}^{-3}$ and central temperatures of $T_{\mathrm{e} 0}=T_{\mathrm{i} 0}=5 \mathrm{keV}$ could be achieved [1]. Furthermore the calculations suggest that, when adding $10 \mathrm{MW}$ of ion cyclotron resonance heating (ICRH) on-axis, the central ion and electron temperatures could be increased to $T_{\mathrm{e} 0}=10 \mathrm{keV}$ and $T_{\mathrm{i} 0}=8 \mathrm{keV}$, respectively. These numbers consider $100 \%$ heating efficiency

\footnotetext{
a See the appendix of Romanelli F. et al 2012 Proc. 24th IAEA Fusion Energy Conf. 2012 (San Diego, CA, 2012) http://www-naweb.iaea.org/napc/ physics/FEC/FEC2012/papers/197_OV13.pdf.
}

which for the case of ion cyclotron resonance frequency (ICRF) heating translates into total absorption of the waves in the bulk plasma (no edge losses). The ICRF heating scenarios available for the hydrogen plasma operation (fundamental majority $\mathrm{H}$ heating and 2 nd harmonic minority ${ }^{3} \mathrm{He}$ heating) have been extensively modelled [2-5] and were experimentally studied a few years ago in dedicated JET experiments [6]. The results indicate poor RF wave absorptivity leading to relatively low heating efficiency $(<50 \%)$ in both cases: fundamental $\mathrm{H}$ majority heating suffers from the well-known unfavourable polarization of the RF fields near the majority cyclotron layer (screening effect) while for the second harmonic ${ }^{3} \mathrm{He}$ heating scenario it is necessary to work at prohibitively high ${ }^{3} \mathrm{He}$ levels to increase the wave absorption.

For the ${ }^{4} \mathrm{He}$ plasmas at $B_{0}=2.65 \mathrm{~T}$ and with similar plasma parameters, fundamental $\mathrm{H}$ minority heating at $f \approx$ $42 \mathrm{MHz}$ will be used. This ICRF heating scheme (either with ${ }^{4} \mathrm{He}$ or more often with D plasmas) is well established and is 
probably the most commonly used in present day machines [7-12]. It has generally a high heating efficiency $(\sim 80 \%)$ and does not require a too precise control of the $\mathrm{H}$ concentration $X[\mathrm{H}]=n_{\mathrm{H}} / n_{\mathrm{e}}$. Moreover, the wave power absorbed by the minority $\mathrm{H}$ ions can in principle be channelled to mainly heat the electrons or the bulk ions, by tuning the $\mathrm{H}$ concentration and/or the plasma density to tailor the RF acceleration and hence the slowing-down of the minority ions onto the bulk plasma particles. In active (D) plasmas, bulk ion heating helps boosting the (D-D) fusion reactivity while central electron heating might be important to keep the core impurity content low in a metallic environment. Although the here reported experiments concern deuterium rather than ${ }^{4} \mathrm{He}$ plasmas (as planned for ITER's non-active phase), theory and experiments show that the RF wave physics governing the minority $\mathrm{H}$ cyclotron absorption and relaxation processes in $\mathrm{D}$ plasmas is very similar to that in ${ }^{4} \mathrm{He}$ plasmas, the main reason being that $\mathrm{D}$ and ${ }^{4} \mathrm{He}$ ions have the same charge-to-mass ratio.

However, two important aspects have to be addressed in more detail to increase the confidence on the performance of this heating scheme in ITER. Here, heating performance is defined as the equilibrium global plasma energy achieved per MW of applied ICRF power and thus includes transport effects as well as radiation losses.

First, it is known that if the $\mathrm{H}$ concentration becomes too large (by e.g. poor wall conditioning or by the use of $\mathrm{H}$ pellet injection for ELM pacing), the performance of the fundamental minority ICRF heating decreases. This is expected by simple RF wave theory due to the change of the RF electric field polarization near the minority ion-cyclotron resonance layer and was confirmed (often unwillingly) in various tokamaks. The rate at which the RF wave absorptivity degrades with the minority concentration depends on several factors (e.g. the machine size, the RF antenna spectrum, the plasma composition, the equilibrium magnetic fields, etc) but for a given tokamak and fixed RF parameters it depends mostly on the plasma density and temperature. In general, higher densities and temperatures favour a slower degradation of the heating efficiency with $X[\mathrm{H}]$, either by directly improving the ion-cyclotron absorption or by enhancing other absorption mechanisms (as e.g. electron Landau damping), which eventually help damping the RF power that is not efficiently absorbed by the minority ions.

The second point concerns the performance of ICRH in general in a full metal environment. In ASDEX-Upgrade (AUG), after the installation of the tungsten wall and divertor, it was verified that the overall ICRF heating performance decreased with respect to the operations with a previously installed carbon wall and that this degradation was associated to large $\mathrm{W}$ levels in the plasma, released from the wall by sheath rectification effects. The heating performance could only be recovered by careful conditioning of the inner wall (boronization) prior to the use of high power ICRF [13]. More recently, JET also started operation with a full metal wall: most parts of the plasma-facing components are made of beryllium whilst the divertor and some recessed main chamber components (as e.g. the NBI shine through plates) are made of tungsten or tungsten-coated carbon [14]. This configuration is very similar to the one that will be used in ITER and hence it is called the ITER-like wall (ILW). Although a considerable increase in the radiation and W levels during ICRH operation is also observed in JET after the ILW installation, the heating performance (plasma energy achieved per MW of ICRF power launched) of the minority H ICRF heating scenario has only slightly decreased with respect to the one observed in the JET carbon fibre composite (JET-C) wall experiments, as will be shown later. Despite the higher radiation, strong central electron heating without significant density increase has been achieved in L-mode and in $\mathrm{H}$-mode plasmas with up to $5 \mathrm{MW}$ of coupled ICRF power. The higher heavy impurity content during ICRF heating leading to higher radiation losses is believed to be related to RF sheath rectification effects, causing sputtering of $\mathrm{Ni}$ and $\mathrm{W}$ in structures magnetically connected to the ICRF antennas. A more detailed analysis revealed that the main chamber $\mathrm{W}$ components and the divertor apron tiles (Wcoated tiles covering the entrance of the divertor) rather than the main part of the divertor (bulk W) are the major source of impurities during high power ICRH, as reported by Bobkov et al [15]. The overall effect of the ILW metallic environment on ICRF heating in JET, including a detailed assessment of the RF-induced heat loads in plasma-facing components, are reported by Jacquet et al in $[16,17]$. The main conclusions are that the higher impurity content w.r.t. the CFC-wall operation does not compromise the performance of ICRF heating too strongly (most of the increased radiation being emitted from the outer part of the plasma [18]) and that the heat loads in the Be limiters close to the RF antennas remain within safe operational limits in all the cases tested. Interestingly, L-mode studies revealed that the ICRF-induced impurity content may strongly depend on the minority $\mathrm{H}$ concentration, as reported by van Eester et al in [19].

In this work dedicated experiments to study the influence of the minority $\mathrm{H}$ concentration on the fundamental ICRF heating performance in conditions similar to those expected in ITER's non-active L-mode operation phase (including the wall and divertor materials) are described. The focus is on the RF heating efficiency and on the fast-particle physics (that controls the RF power eventually absorbed by the bulk plasma by collisions with the fast minority ions) since a detailed analysis of the plasma-wall interactions and the RF-induced impurity content comprising their dependence on the minority $\mathrm{H}$ concentration is already given in [15-19].

The paper is divided as follows. In section 2 the main experimental results are presented, including the dependence of the global plasma parameters (energy, temperature, etc), the $\mathrm{RF}$ heating efficiency and the fast-particle dynamics on the $\mathrm{H}$ concentration. In section 3, numerical RF wave and FokkerPlanck tools [20-23] are validated against the experimental data. In section 4, the same codes are used to assess the heating performance of the fundamental $(\mathrm{H})-{ }^{4} \mathrm{He}$ minority ICRH scheme in ITER's half-field phase and a summary is given in section 5 .

\section{Experimental observations}

The time traces of several quantities for three similar Lmode discharges with different hydrogen concentrations are shown in figure 1: (a) ICRF power, $(b)$ core line integrated density, $(c)$ hydrogen concentration, $(d)$ total radiated power, $(e)$ central electron temperature and $(f)$ plasma stored energy. 

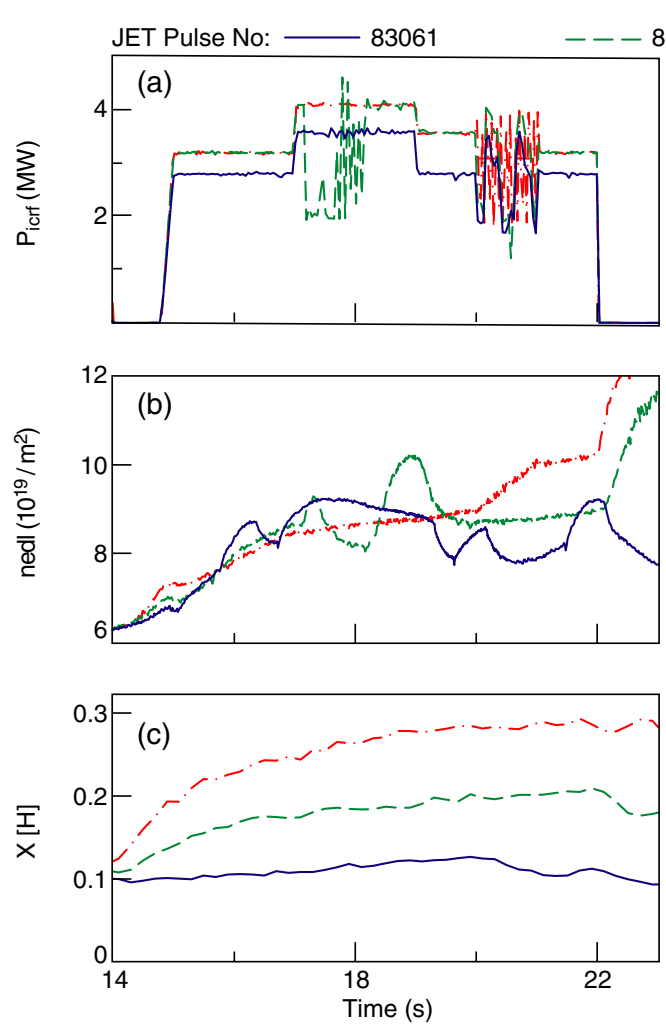
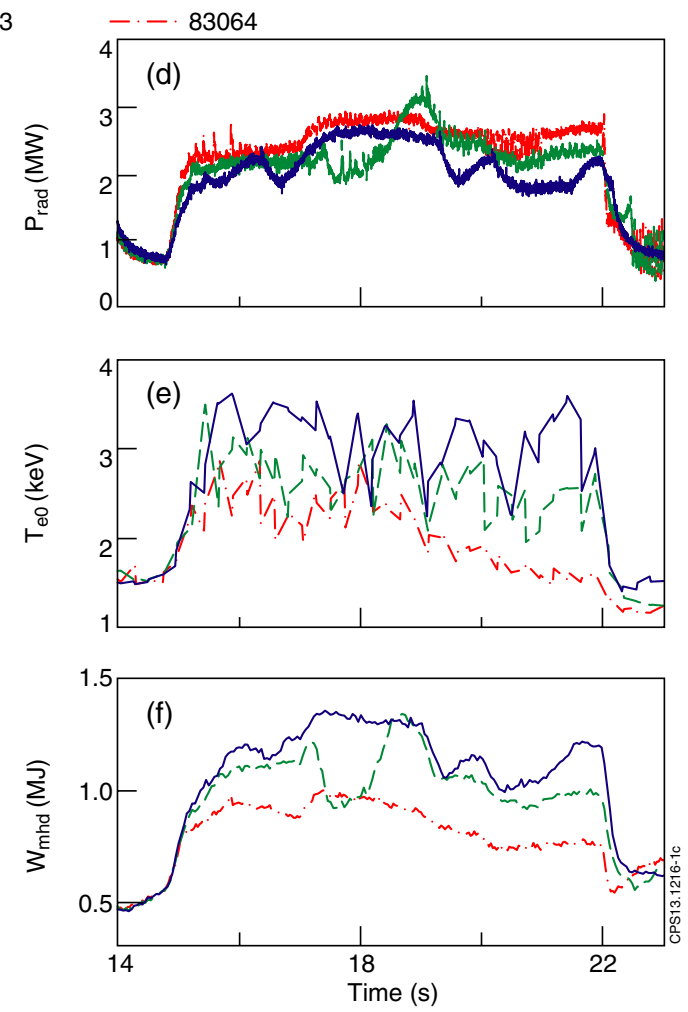

Figure 1. Time traces of various signals for three similar JET discharges: $(a)$ ICRF power, $(b)$ line averaged electron density, $(c)$ hydrogen concentration, $(d)$ total radiated power, $(e)$ central electron temperature and $(f)$ plasma stored energy.

The discharges were performed at $B_{0}=2.7 \mathrm{~T}$ and $I_{\mathrm{P}}=$ 2.0 MA, low triangularity and low antenna-plasma distance $(\sim 10-11 \mathrm{~cm}$ in the mid-plane) to favour efficient ICRF coupling. The RF frequency was $f=42 \mathrm{MHz}$ (placing the $\mathrm{H}$ ion-cyclotron resonance roughly on-axis) and dipole phasing ( $\pi$ phasing between adjacent antenna straps) was adopted. The $\mathrm{H}$ concentration was varied from $5-30 \%$ by injecting pure $\mathrm{H}_{2}$ from a dedicated divertor gas injection module in addition to the standard $\mathrm{D}_{2}$ feedback controlled injection based on the core plasma density request. The $\mathrm{H}_{2}$ injection waveform was tailored to provide roughly constant $X[\mathrm{H}]$ during the discharges and no evidence of $\mathrm{H}$ accumulation was observed when the $\mathrm{H}$ content was varied from shot to shot, highlighting the low retention and recycling properties of the new Be wall [24]. The $\mathrm{H}$ concentration was measured both by edge spectroscopy (ratio of $\mathrm{H}_{\alpha} / \mathrm{D}_{\alpha}$ Balmer lines) and by mass spectrometry with a good agreement for $X[\mathrm{H}]>$ $3-4 \%$. Unless otherwise mentioned, the values quoted here correspond to the latter and the measured concentrations are considered to also represent the core $X[\mathrm{H}]$ values.

The rapid variations of the plasma density observed in figure $1(b)$ are not due to the $\mathrm{H}_{2}$ injection but rather due to the transition of the plasma to a modest confinement $\mathrm{H}$-mode state, referred to as M-mode [25]. The impact of increasing the $\mathrm{H}$ concentration on the plasma performance is clear: both the plasma stored energy $(f)$ and the central electron temperature (e) are lower when more $\mathrm{H}_{2}$ is injected with similar amounts of ICRF power applied. The total radiation $(d)$ is comparable in the three discharges, being more influenced by the density and/or by the transitions of the plasmas into the M-mode than by the $\mathrm{H}$ concentration.
In figure 2 , the averaged values of $(a)$ plasma stored energy, $(b)$ central electron temperature, $(c)$ total radiated power and $(d)$ bulk radiated power reached during ICRH are plotted as a function of the $\mathrm{H}$ concentration. The data correspond to $0.2 \mathrm{~s}$ time averaged values of the measurements taken in a series of L-mode discharges similar to the ones shown in figure 1 . The central densities are within $n_{0}=$ $3.5-4.0 \times 10^{19} \mathrm{~m}^{-3}$ and data points with ICRF power between 3.0 MW (circles) and 3.5 MW (squares) were included to have a better statistics. The large error bars on the central electron temperature data $(b)$ are due to the averaging of the $T_{\mathrm{e}}$ time traces over one or more sawtooth periods.

The deleterious effect of increasing the minority concentration on the ICRF heating performance is clear in figures $2(a)$ and $(b)$. Interestingly, the plasma thermal energies reached for a given amount of RF power are not degraded below $X[\mathrm{H}]<15 \%$, at which point they start to decrease with $X[\mathrm{H}]$ reaching about $2 / 3$ of the original values at $X[\mathrm{H}]=30 \%$. The central electron temperatures, on the other hand, show a systematic (linear) reduction with $X[\mathrm{H}]$ already from very low $\mathrm{H}$ concentrations: values of $T_{\mathrm{e}}=4.5 \mathrm{keV}$ are achieved at low $\mathrm{H}$ concentrations and are reduced by a factor of $2-3$ when $X[\mathrm{H}]=30 \%$ is reached. This different behaviour comes from the fact that, while both the energies and electron temperatures reached during ICRH are functions of the RF heating efficiency (and transport), the latter also depends on the slowing-down of the fast ions, which is strongly modified when the $\mathrm{H}$ concentration is increased, as will be shown later. Hence, the $\sim 30 \%$ decrease of $T_{\mathrm{e} 0}$ observed for $X[\mathrm{H}]<15 \%$ (where the thermal energies are roughly constant) is less strongly related to the RF heating efficiency than to the reduction of the fast ion 

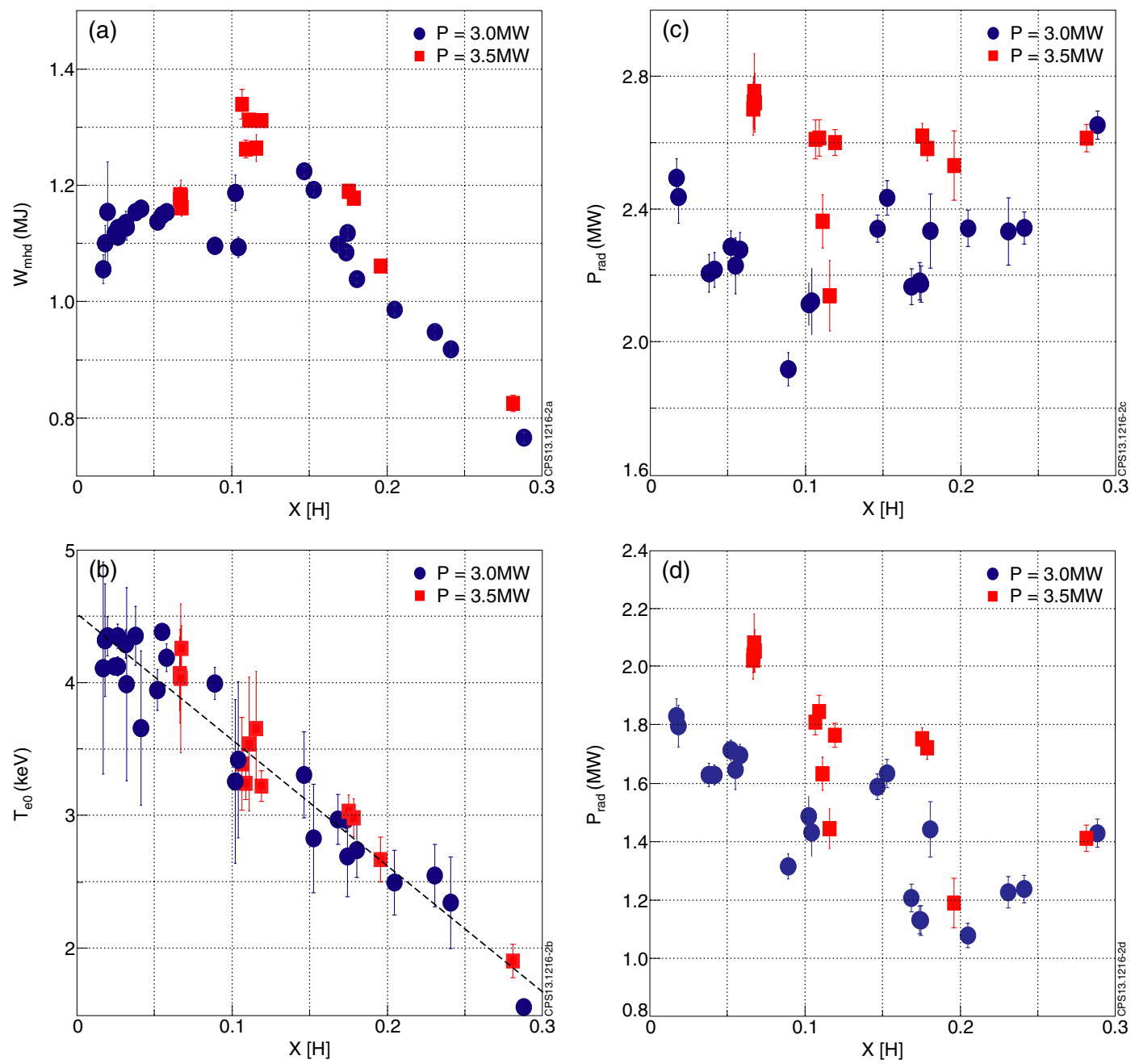

Figure 2. Average values of several quantities as function of the H concentration for two ICRF power levels in similar L-mode JET discharges. (a) Plasma stored energy, $(b)$ central electron temperature, $(c)$ total radiated power and $(d)$ bulk radiated power (inside the last closed flux surface). The measured quantities are averaged over $0.2 \mathrm{~s}$.

tails with $X[\mathrm{H}]$ and the consequent re-channelling of a fraction of the RF wave energy to the bulk D ions. Unfortunately, ion temperature measurements were not available in these experiments to corroborate this observation.

In figure $2(c)$ the total radiated power measured by bolometry is shown as a function of $X[\mathrm{H}]$ while in figure $2(d)$ the fraction radiated in the bulk plasma is depicted. Despite the relatively large spread in the data, one sees that the total radiation is not very sensitive to the $\mathrm{H}$ concentration but that the bulk radiation decreases from slightly below $2 \mathrm{MW}$ at low $X[\mathrm{H}]$ values to $\sim 1.2 \mathrm{MW}$ at $X[\mathrm{H}]=20 \%$ and starts to increase afterwards. A more detailed analysis revealed that the decrease of the bulk plasma radiation is related to a reduction of the high$\mathrm{Z}$ impurity content ( $\mathrm{Ni}$ and $\mathrm{W}$ ) measured in the plasma core when the $\mathrm{H}$ concentration is increased [19], despite the higher edge losses which are related to poorer RF wave absorption leading enhanced plasma-wall interaction. Unfortunately the edge properties of these plasmas also changed during the $X[\mathrm{H}]$ scan making it difficult to conclude if the lower impurity level observed at moderate $\mathrm{H}$ levels is related to a core (RF absorption) or to an edge (plasma-wall interaction) effect. It is worth mentioning that the total radiation in these discharges represents a large fraction of the auxiliary power applied
$\left(P_{\text {rad }} / P_{\text {icrf }} \approx 60-70 \%\right)$. These figures are considerably higher than the ones seen with the carbon wall in similar plasma conditions (30-40\%) and as mentioned before, are caused by the additional influx of tungsten in ICRF heated discharges due to RF sheath induced sputtering of $\mathrm{W}$ elements in the main chamber and upper parts of the divertor $[15,17,26]$. The same effect was already observed in JET-C discharges but Ni coming from structures near the ICRF antennas was identified as the main metallic impurity at that time [27].

Despite the different radiation losses, the energies achieved per MW of ICRF power in similar conditions in the JET-C and JET-ILW experiments are comparable, provided the edge plasma density is not too low [15]. An example is given in figure 3 , where the equilibrium values of the stored plasma energy reached as a function of the ICRF applied power in a series of JET-ILW L-mode discharges with $X[\mathrm{H}]<15 \%$ (including a subset of the data shown in figure 2) are compared with the energies reached in similar JET-C plasmas.

The global RF heating performance, defined as $\left(W_{\text {mhd }}-W_{\text {mhd, } 0}\right) / P_{\text {icrf }}$, where $W_{\text {mhd, } 0} \approx 0.5 \mathrm{MJ}$ represents the stored energy in the purely ohmically heated phase of the discharges $\left(P_{\mathrm{ohm}} \approx 1.5 \mathrm{MW}\right.$ for the considered $I_{\mathrm{P}}=2 \mathrm{MA}$ plasmas) is about $0.23 \mathrm{MJ} \mathrm{MW}^{-1}$ for the JET-ILW pulses, 


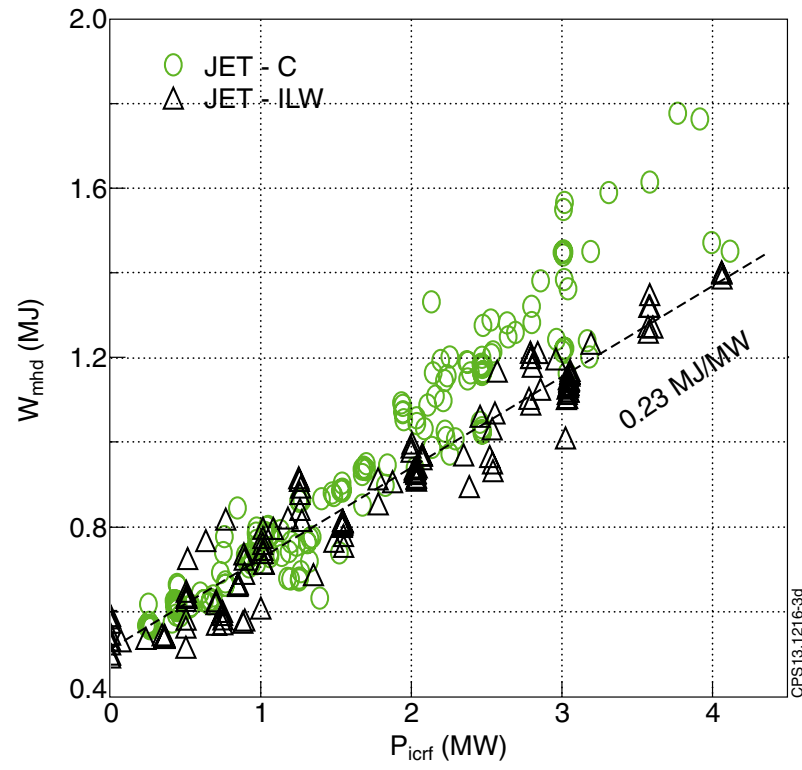

Figure 3. Plasma stored energies $\left(W_{\text {mhd }}\right)$ achieved as a function of the ICRF power for similar L-mode discharges in JET-ILW and JET-C with $B_{0}=2.7 \mathrm{~T}, I_{\mathrm{P}}=2 \mathrm{MA}$ and $f=42 \mathrm{MHz}$. All the data points correspond to $X[\mathrm{H}]<15 \%$.

around $20 \%$ lower than the value obtained in JET-C at high ICRF power. Note that this quantity represents the equilibrium energy reached per MW of RF power applied to the plasma and thus includes transport and radiation losses. As discussed in $[17,18]$, the somewhat lower performance seen in JETILW when the ICRF power is raised above $2 \mathrm{MW}$ is due to the higher radiation losses observed with the new metallic wall during RF operation but it still represents a very modest reduction if one realizes that the total radiation has increased by a factor of more than two when similar ICRF power levels are applied [17].

In figure 4 , the ICRF heating efficiencies $\eta=P_{\text {abs }} / P_{\text {icrf }}$, obtained by break-in-slope (BIS) analysis of the plasma energy response to sharp ICRF power steps [28] applied to the discharges discussed in figure 2 are plotted as a function of the $\mathrm{H}$ concentration. Unlike the global RF heating performance discussed earlier, the RF heating efficiency inferred from BIS analysis of the plasma energy response $\left(P_{\mathrm{abs}} \sim \partial W / \partial t, \partial t \rightarrow\right.$ 0 ) does not depend on the plasma confinement nor on the radiation losses as long as a short enough time interval is taken for estimating the time derivatives of the energy signal. Thus, it reflects the instantaneous character of the RF wave absorption rather than the fate of the absorbed power after transport and radiation losses take over. Fast ion dynamics is captured to a certain degree by the BIS analysis since the slowing-down time of the minority $\mathrm{H}$ ions is typically much shorter than the energy confinement time of the bulk particles in the studied discharges.

Nevertheless, a strict relation between the instantaneous heating efficiency and the plasma energy values achieved as a function of $X[\mathrm{H}]$ (figure $2(a)$ ) is clearly observed: the heating efficiency is roughly constant between $\eta=0.6-0.8$ up to $X[\mathrm{H}]=15 \%$ and then starts to decrease, reaching $\eta \sim 0.4$ at $X[\mathrm{H}]=30 \%$. This suggest that from the RF point of view, it could be beneficial to work at increased $\mathrm{H}$ concentrations

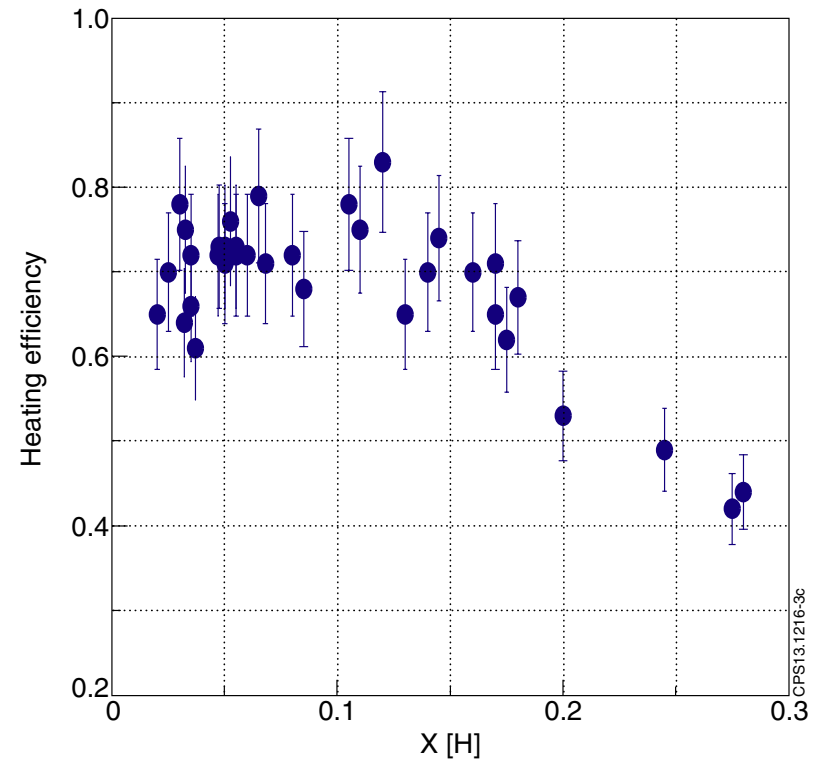

Figure 4. RF heating efficiency $\left(P_{\mathrm{abs}} / P_{\text {icrf }}\right)$ as a function of $\mathrm{H}$ concentration inferred from BIS analysis of the plasma stored energy $\left(W_{\text {mhd }}\right)$ evolution during ICRF power steps applied to similar JET-ILW L-mode discharges $\left(B_{0}=2.7 \mathrm{~T}, f=42 \mathrm{MHz}\right)$.

$(\sim 15 \%)$ to profit from the reduced radiation and impurity levels as shown in figure $2(d)$, at least in dominantly ICRF heated discharges.

The strong correlation between the dependencies of the RF heating efficiency (figure 4) and of the global heating performance (figure $2(a)$ ) with $X[\mathrm{H}]$ suggests that the degradation of the ICRF power absorption rather than changes in transport or radiation is the main responsible for the lower heating performance observed at high $X[\mathrm{H}]$ in the reported experiments. Transport simulations of these discharges also confirmed that the global transport properties of the plasma are not strongly altered by the change in the $\mathrm{H}$ concentration for $X[\mathrm{H}]<25 \%$.

The impact of the $\mathrm{H}$ concentration on the fast-particle dynamics during ICRH was studied with the horizontal neutral particle analyser (NPA) [29]. In figure 5 (left), time traces of various signals in three similar JET discharges with $3 \mathrm{MW}$ of ICRF heating are shown: $(a) \mathrm{H}$ concentration, $(b)$ neutral flux for $200 \mathrm{keV} \mathrm{H}$ ions and $(c)$ central electron temperature. As expected, the number of counts registered by the NPA at high energies decreases when the $\mathrm{H}$ concentration is increased indicating a smaller population of supra-thermal ions. This is a well-known phenomenon and is simply related to the fact that for higher $X[\mathrm{H}]$ values, more $\mathrm{H}$ ions have to share the same amount of RF power that is absorbed in the plasma and hence the power per particle (and thus its acceleration) is reduced. The drop of the fast ion content (effective energy and population) is also confirmed by the reduction of the sawtooth activity for higher $X[\mathrm{H}]$ as illustrated by the central $T_{\mathrm{e}}$ signals (c), since larger fast ion pressures within the $q=1$ surface tend to stabilize the kink instability leading to longer sawtooth periods in ICRF heated discharges than in non-heated ones (see [30] for more details on this mechanism).

In figure $5(d)$ the NPA energy spectra of the fast $\mathrm{H}$ tails are shown for the three discharges represented on the 

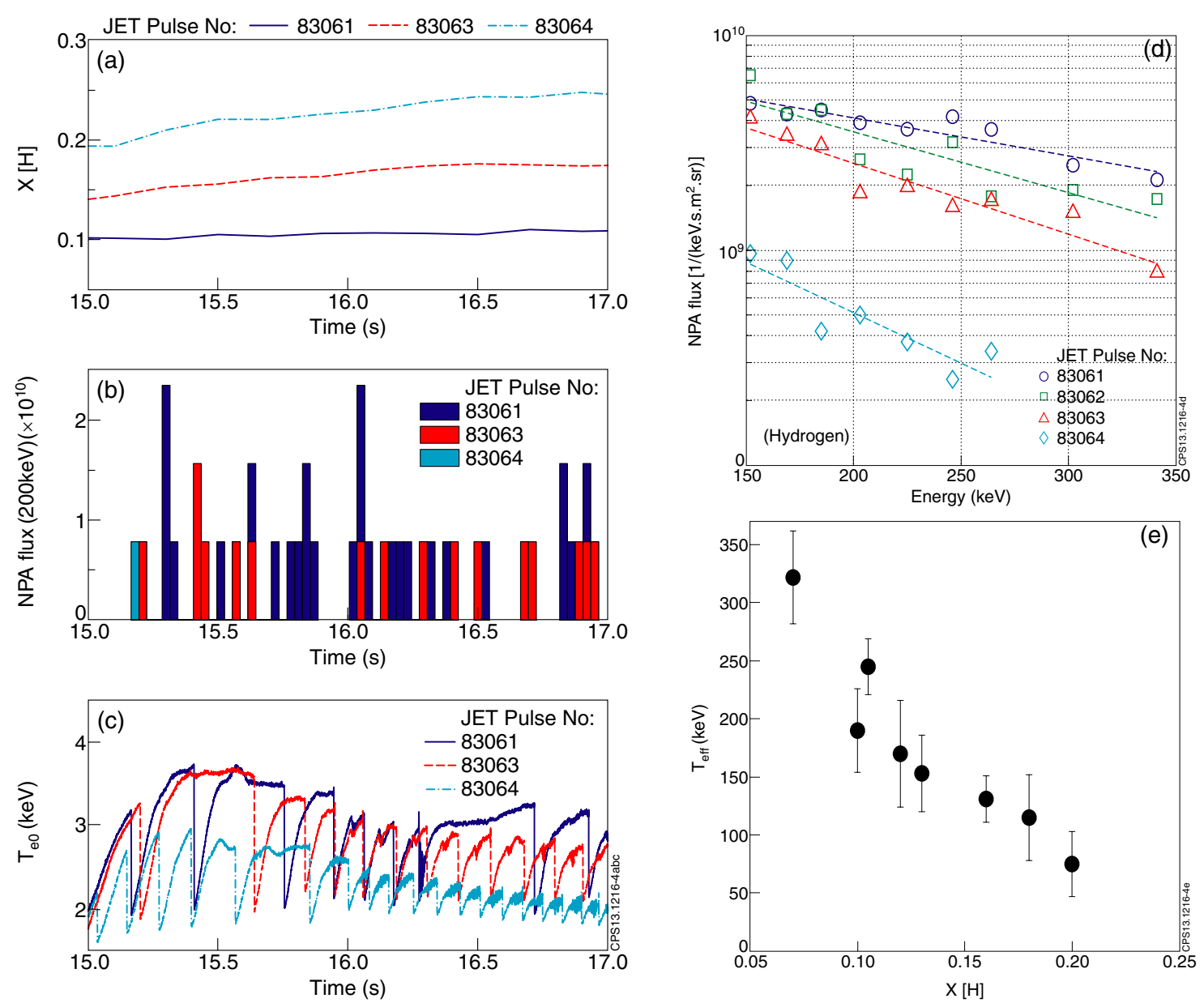

Figure 5. Left: time traces of various signals in three similar JET discharges: $(a) \mathrm{H}$ concentration, $(b) \mathrm{NPA}$ flux for $200 \mathrm{keV} \mathrm{H}$ ions and $(c)$ central electron temperature from ECE radiometer. Right: $(d)$ NPA spectra for different $\mathrm{H}$ concentrations and $(e)$ effective temperature of the fast $\mathrm{H}$ ions inferred from NPA data slopes as a function of the $\mathrm{H}$ concentration. All the data correspond to a similar plasma densities, $n_{\mathrm{e}} \mathrm{d} l=(8.5 \pm 1.0) \times 10^{19} \mathrm{~m}^{-2}$ and ICRF power, $P_{\text {icrf }}=3.2 \pm 0.2 \mathrm{MW}$.

right. An additional pulse with $X[\mathrm{H}]=12-15 \%$ (pulse $83062)$ is also included. The data correspond to $2 \mathrm{~s}$ time averages taken in similar plasmas with $P_{\text {icrf }}=3 \mathrm{MW}$. The high concentration data (pulse 83064) are only plotted for illustrations purposes but cannot be considered for a deeper analysis, since the NPA counts become too scarce and fluxes below $10^{9}\left[1 /\left(\mathrm{keV} \mathrm{s} \mathrm{m}^{2} \mathrm{sr}\right)\right]$ are within the background level of the measurements. In figure $5(e)$ the effective temperatures of the $\mathrm{H}$ tails with $150 \mathrm{keV}<E<350 \mathrm{keV}$ estimated from the slopes of the NPA spectra (as shown in figure $4(d)$ ) are plotted as a function of the $\mathrm{H}$ concentration. These estimates should be interpreted as the maximum averaged energy the $\mathrm{H}$ ions have achieved in the (horizontal) line-of-sight of the detector, namely in the region where the RF power absorption is maximum. The $X[\mathrm{H}]>20 \%$ data points were discarded since the NPA statistics is too poor. The $T_{\text {eff }}$ values representing the $\mathrm{H}$ ion tail within $150-350 \mathrm{keV}$ decrease by a factor of roughly 3 when $X[\mathrm{H}]$ is increased from $10 \%$ to $20 \%$. Again, this effect is a combination of the heating efficiency degradation (lower power density values) and the increase of the number of absorbing minority ions (less power per particle), leading to weaker RF acceleration. Furthermore, the fact that the plasma collisionality is higher at large $X[\mathrm{H}]$ (lower $T_{0}$ ) implies, by itself, a reduction in the minority tail formation $\left(T_{\mathrm{eff}} \propto T_{\mathrm{e}}^{3 / 2}\right)$.
Competition with direct electron absorption (either by fast wave Landau damping or eventually by mode conversion) may also play a role when the minority concentration is high, but the experiments as well as theoretical predictions indicate that this effect is relatively small in the studied $X[\mathrm{H}]$ range.

\section{RF modelling of the JET discharges}

The $(\mathrm{H})-\mathrm{D}$ ICRF heating experiments reported here were modelled using various numerical tools. In figure 6 , the power absorption profiles for the various plasma species obtained with the 2D full-wave CYRANO code [20,21] $((a),(b))$ are compared with the respective profiles obtained with the 1D TOMCAT wave solver [22] $((c),(d))$ for two different $\mathrm{H}$ concentration levels. The CYRANO curves represent the actual flux surface averaged absorbed power density profiles $\left(\mathrm{MW} \mathrm{m}^{-3}\right.$ ) normalized to a total absorbed power of $1 \mathrm{MW}$ while the TOMCAT curves represent the local absorbed power (W) in a finite element width for launched power of $1 \mathrm{~W}$. All the simulations were done with RF frequency $f=42 \mathrm{MHz}$, toroidal wave number $N_{\phi}=27$ (dominant mode for dipolephasing excitation), $B_{0}=2.7 \mathrm{~T}$ and main plasma profiles similar to those found in the experiments for the low $\mathrm{H}$ 

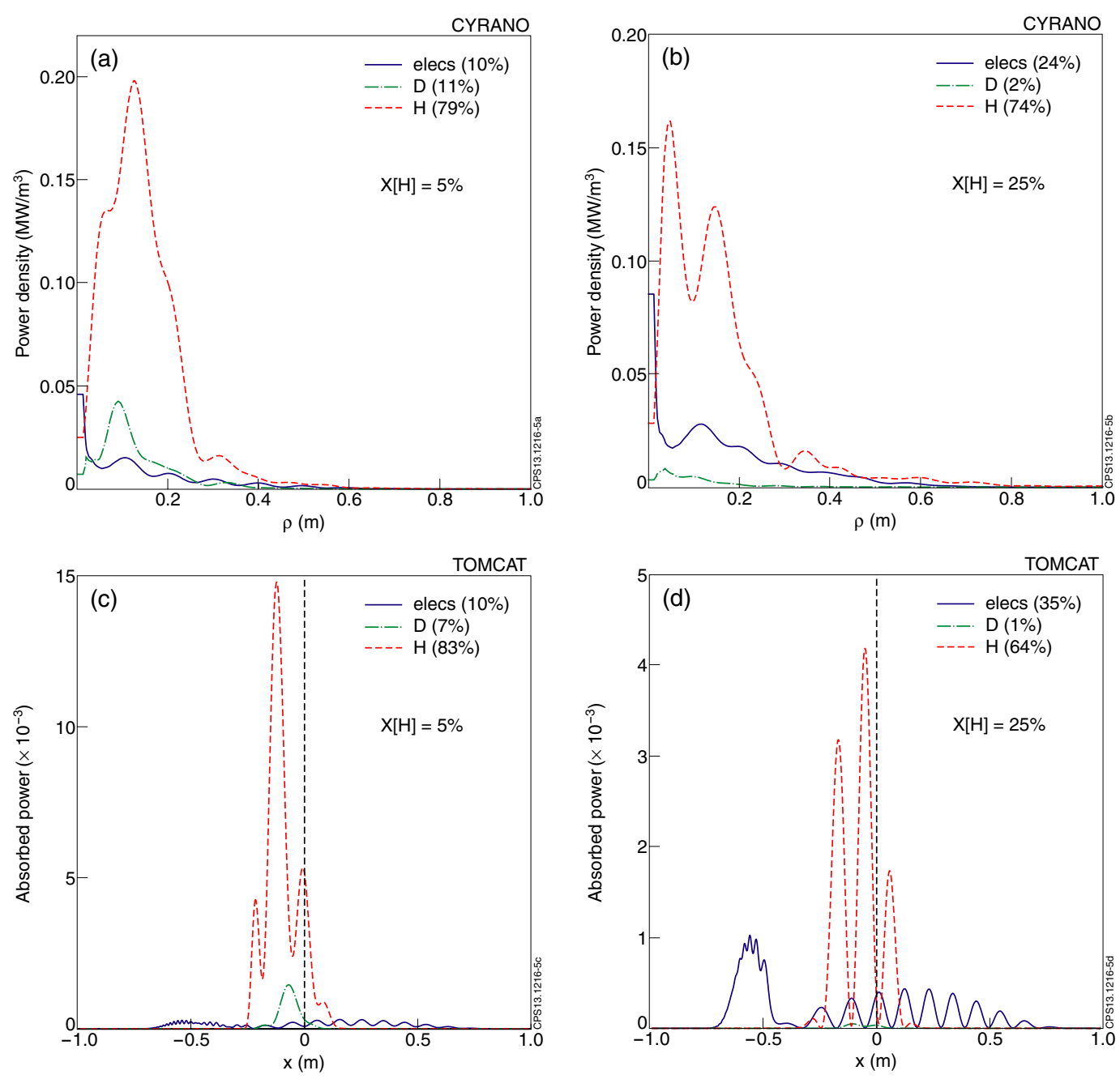

Figure 6. Power absorption profiles calculated with the 2D CYRANO full-wave code $(a),(b)$ and with the $1 \mathrm{D}$ TOMCAT wave solver $(c)$, $(d)$ for two H concentration values for the (H)D ICRF heating scheme in JET: $X[\mathrm{H}]=5 \%$ and $X[\mathrm{H}]=25 \%$. The abcissae represent the minor radius $\rho$ of the flux surfaces in the CYRANO case and the equatorial coordinate $x=R-R_{0}$ in the TOMCAT case.

concentration cases $\left(n_{0}=3.5 \times 10^{19} \mathrm{~m}^{-3}, T_{\mathrm{e} 0}=T_{\mathrm{i} 0}=\right.$ $4 \mathrm{keV}$ ). The effect of the plasma temperature on the RF power absorption (also observed experimentally) will be discussed later. All species are considered Maxwellian-which is a good approximation in the case of fundamental ICRF heatingand the temperature of the minority ions is kept at thermal values. Despite the different models adopted in the two codes and the fact that the 1D TOMCAT code by definition ignores poloidal geometric effects that might be crucial, their results are qualitatively similar: at low $X[\mathrm{H}]$, ion-cyclotron absorption of the minority $\mathrm{H}$ is by far the dominant process while for higher concentrations this process loses strength and electron Landau damping starts to play an important role in the wave absorption. Note that the TOMCAT simulations start to show a hint of mode conversion to the Bernstein wave in the high concentration case, manifested as a localized electron absorption lobe on the high-field side of the plasma. This effect is not seen in the CYRANO simulations for the numerical grid adopted in these simulations leading to smaller electron power fractions predicted at high $X[\mathrm{H}]$. In any case, short wavelength modes excited in the mode conversion region violate the criterion for finite Larmor radius expansion $k_{\perp} \rho_{\mathrm{L}} \ll 1$ (which is used in both codes) and thus have to be interpreted carefully: the actual width of the short wave absorption is probably overestimated but the amount of power absorbed by mode conversion $(\sim 15 \%)$ is properly accounted for, as indicated by benchmarking with an integro-differential version of the TOMCAT code [31].

Being a 1D wave solver, TOMCAT has the capability of estimating the single-pass absorption (SPA) of the RF wave when it crosses the plasma. In this particular case the highfield side cut-off of the fast wave is inside the plasma so that the waves that are not fully absorbed in the plasma core and reach this point are reflected back towards the low-field side. In these cases, the SPA refers to a full (double) transit of the waves in the plasma and is inferred from the remaining active power (Poynting flux) left with the wave after it is bounced back to the antenna.

As mentioned, the wave absorptivity (or SPA) depends on various plasma parameters and in particular on the plasma temperature. On the other hand, because in the described experiments ICRH was the only auxiliary power source, the actual plasma temperatures reached depend on the wave absorptivity itself and the accurate way of 

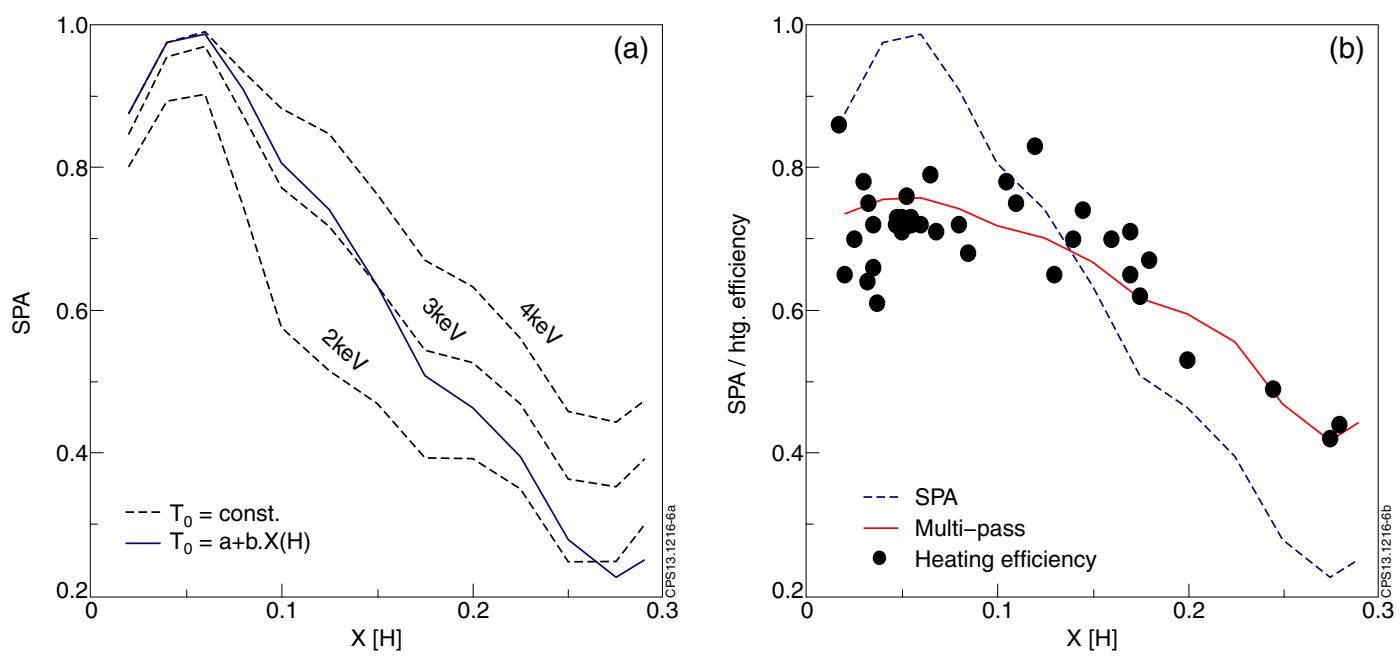

Figure 7. (a) SPA calculated by the 1D TOMCAT code as a function of the $\mathrm{H}$ concentration for the JET (H)D scenario for fixed temperature values (dotted) and for a linear decay of the plasma temperature (solid) based on the experimental findings. (b) Comparison between the theoretical heating efficiency (using a multi-pass model with $23 \%$ losses per wave transit) and the experimental values inferred by BIS analysis of RF transients in the experiments (figure 4).

studying this problem would be to perform self-consistent wave-transport simulations of these discharges. However, since the experimental $T_{\mathrm{e}}$ values are known, we will adopt a simpler approach and compute the single-pass wave absorption as a function of $X[\mathrm{H}]$ by correcting the temperatures based on the experimental data. For this, the electron (and ion) temperatures were approximated as a linear function of the form $T=a+b \cdot X[\mathrm{H}]$, where $a$ and $b$ are obtained from fitting the experimental $T_{\mathrm{e}}$ data, as shown in figure $2(b)$. Unfortunately ion temperature measurements were not available in the experiments and the $T_{\mathrm{e}}=T_{\mathrm{i}}$ assumption may be inaccurate in these rather low density plasmas, in particular at high $X[\mathrm{H}]$ values for which the collisional relaxation of the minority $\mathrm{H}$ is predominantly onto the bulk ions.

The simulation results are illustrated in figure 7(a), where the SPA of the RF waves calculated by TOMCAT are plotted as a function of the $\mathrm{H}$ concentration for three fixed temperatures (dotted) and for the linearly corrected temperature values (solid). It can be seen that when the actual temperature drop is included, the SPA for high $X[\mathrm{H}]$ values is only about 0.2 , about 2 times lower than the value obtained without the $T_{\mathrm{e}}$ correction. In the medium $X[\mathrm{H}]$ range $(\sim 15 \%)$, the SPA varies from 0.4 to 0.8 depending on the temperature considered while at low concentrations the absorptivity is less sensitive to the plasma temperature.

In reality, the low absorption waves are not 'reabsorbed' by the antenna after a double-pass but are again reflected back from the low-field side cut-off and ultimately from the metallic wall, and thus keep sloshing around the plasma until they have damped all their energy. However, on top of the edge losses that occur the first time the launched waves cross the scrape-off layer (also present in the full SPA cases), the low absorptivity waves lose part of their energy every time they come close the evanescence region. By adopting a multi-pass model that accounts for the (a priori unknown) losses per transit, it is possible to estimate the theoretically expected heating efficiency of an ICRF scenario by considering a large enough number of transits for the wave power to be fully damped. This method can also be used the other way around, i.e. to estimate the RF wave losses based on the experimental values of the heating efficiencies and the predicted SPA curves for the experimental plasma conditions. This is illustrated in figure $7(b)$, where the theoretical heating efficiencies (solid) obtained with a multi-pass model using the temperature dependent SPA curves calculated with TOMCAT (figure $7(a)$ ) are adjusted to the experimental heating efficiency values. The best fit was obtained with $23 \%$ losses per transit as seen in the $X[\mathrm{H}] \approx 0.05$ data, where the SPA is close to unity. If the plasma would have been kept at $T=4 \mathrm{keV}$ by compensating the lower ICRF heating efficiency with other heating method, the expected heating efficiency at high $X[\mathrm{H}]$ would increase from 0.4 to approximately 0.6 .

As a final modelling exercise, a 1D isotropic FokkerPlanck solver [23] based on the Stix-Karney formalism $[32,33]$ was used to interpret the dependence of the fastparticle dynamics on the $\mathrm{H}$ concentration observed with the NPA during the experiments. For a given $\mathrm{H}$ concentration, the maximum value of the power density profile computed by the 2D CYRANO wave code for $3 \mathrm{MW}$ of RF power and normalized according to the heating efficiency estimated experimentally (figure 4) was used as the source term for the FP calculations. This means that the fast $\mathrm{H}$ distributions computed reflect the most energetic $\mathrm{H}$ ions present in the plasma, namely close to the ion-cyclotron resonance position. In this work no attempt is made to compute the distributions in several plasma radii and try to synthetically mimic the (integrated) NPA measurements. The RF field components and the value of the perpendicular wave vector $k_{\perp}$ at the surface of maximum power absorption were also imported from the CYRANO calculations. Because the $\mathrm{H}$ concentrations reach large values, the self-collisions between the accelerated $\mathrm{H}$ ions cannot be neglected and an iterative procedure is used to evolve the accelerated $\mathrm{H}$ distributions towards a steady state [23]. The power absorbed by the background plasma species (deuterons and electrons) was neglected and their distributions were 

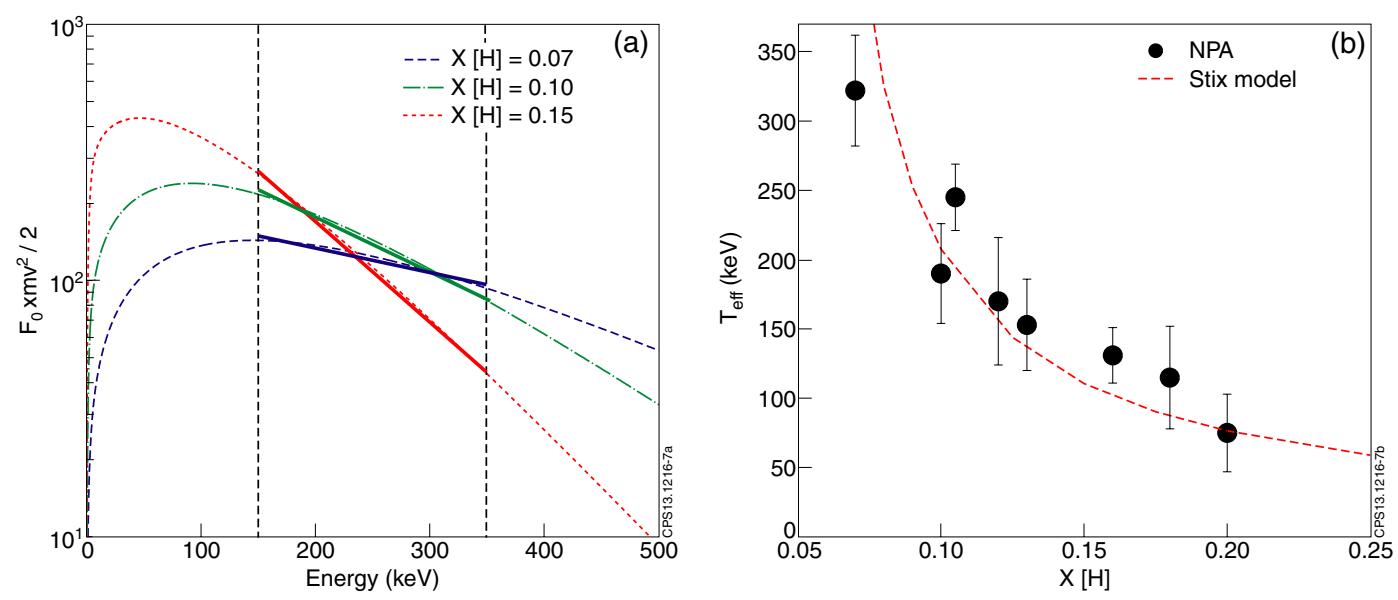

Figure 8. (a) Energy distributions $\left(F_{0} \times m_{\mathrm{H}} v^{2} / 2\right)$ computed for the $\mathrm{H}$ minority for $P_{\text {icrf }}=3 \mathrm{MW}$ and three hydrogen concentration values: $X[\mathrm{H}]=7 \%$ (dashed), $X[\mathrm{H}]=10 \%$ (dashed-dotted) and $X[\mathrm{H}]=15 \%$ (dotted). $(b)$ Comparison between the effective tail temperatures obtained as a function of $X[\mathrm{H}]$ by the Fokker-Planck simulations and from the NPA data by using the same energy window (150-350 keV) for inferring $T_{\text {eff }}$ from the logarithmic slopes of the local energy distributions.

kept Maxwellian throughout the iterative loop, with linearly decreasing temperatures given by $T_{\mathrm{e}}=T_{\mathrm{D}}=a+b \cdot X[\mathrm{H}]$, as described before.

In figure $8(a)$ the hydrogen energy distributions $F_{0} \times$ $m_{\mathrm{H}} v^{2} / 2$ obtained by the Fokker-Planck code for $X[\mathrm{H}]=$ $7 \%, X[\mathrm{H}]=10 \%$ and $X[\mathrm{H}]=15 \%$ are plotted in logscale as a function of the $\mathrm{H}$ ion energy. For a qualitative comparison with the NPA data, the effective temperatures of the ion tails in the simulation were defined in terms of the local (logarithmic) slopes of the energy distribution over an energy range comparable to the NPA one (150-350 keV) rather than integrating over the whole slowing-down distribution energies. The effective temperatures obtained by the FP modelling are compared with the experimental values from the NPA in figure $8(b)$. It is surprising to see that despite the crudeness of the FP model and the fact that no efforts were made to quantitatively mimic the diagnostics characteristics (such as the line-of-sight, the phase-space response, etc) the simulations describe the mitigation of the fast $\mathrm{H}$ tail with $X[\mathrm{H}]$ satisfactorily. It has to be said that at lower concentrations (not shown) the simulations give considerably higher effective energy levels than the NPA measurements.

\section{Fundamental $(\mathrm{H})-{ }^{4} \mathrm{He}$ ICRH performance in ITER}

In this section we extend the numerical modelling validated in the JET experiments to the $\mathrm{H}$ minority ICRH scheme in ITER half-field ${ }^{4} \mathrm{He}$ plasmas. For this, we consider the plasma equilibrium parameters described in [1]: $B_{0}=2.7 \mathrm{~T}$, $I_{\mathrm{P}}=7.5 \mathrm{MA}$, L-mode profiles with $n_{0}=3.5 \times 10^{19} \mathrm{~m}^{-3}$ and temperatures varying between $T_{\mathrm{e} 0}=T_{\mathrm{i} 0}=3 \mathrm{keV}$ and $8 \mathrm{keV}$, ITER's reference being $T_{0}=5 \mathrm{keV}$. The RF frequency is $f=42 \mathrm{MHz}$ and different antenna phasings available in ITER were considered. The results are summarized in figure 9 , where the SPA curves (left) and the corresponding heating efficiencies (right) are shown as a function of the $\mathrm{H}$ concentration $X[\mathrm{H}]$ for two cases: $(a),(c)$ different temperatures and $00 \pi \pi$ $\left(N_{\phi}=27\right)$ phasing and for $(b)$ fixed temperature $\left(T_{0}=5 \mathrm{keV}\right)$ but different antenna phasings. For the latter, only the main toroidal mode numbers $N_{\phi}$ of the spectra excited by three dipole-phasing configurations available in ITER as computed by the ANTITER-II code [34] were used: $00 \pi \pi\left(N_{\phi}=27\right)$, $0 \pi \pi 0\left(N_{\phi}=39\right)$ and $0 \pi 0 \pi\left(N_{\phi}=63\right)$. The expected heating efficiencies $\eta_{\text {theo }}(c),(d)$ were estimated using a multi-pass model with the same $(23 \%)$ wave losses per transit in the plasma as obtained from the analysis of the JET discharges. This choice is obviously a too simplistic approximation and is only based on the fact that the RF settings and the wall materials are similar in the two cases. Mode-conversion effects were negligible throughout the $X[\mathrm{H}]$ range studied.

The beneficial effect of pre-heating the plasma on the wave absorption is clear in figures $8(a)$ and $(c)$. For the reference ITER value of $T_{0}=5 \mathrm{keV}$ the heating efficiency remains above $\eta_{\text {theo }} \sim 0.5$ for most of the $X[\mathrm{H}]$ values studied and the RF heating scenario is expected to yield good plasma heating even if high levels of $\mathrm{H}$ are present. If the plasma is too cold $\left(T_{0}<3 \mathrm{keV}\right)$ and the $\mathrm{H}$ concentration too high, the heating efficiency will be reduced. Alternatively, if an antenna phasing that excites a spectrum with higher $k_{\|}=N_{\phi} / R$ power lobes is used, the heating efficiency at large $X[\mathrm{H}]$ can be enhanced. However, higher $k_{\|}$phasings feature lower antenna loading resistance and a compromise between keeping a good coupling while heating the plasma more efficiently has to be considered for optimizing the ICRH performance [35].

The increase of the RF heating efficiency with $T_{0}$ and $k_{\|}$is mainly related to the increased width of the cyclotron absorption region in these conditions (IC width $\propto k_{\|} v_{\mathrm{T}}$ ), leading to enhanced Doppler-shifted power absorption of the minority ions around the cold IC resonance layer. Aside from the plasma temperature and the antenna spectrum, the machine size also plays an important role on the influence of the $\mathrm{H}$ concentration on the SPA of the RF waves in the plasma. In larger tokamaks the ratio between the perpendicular wavelength and the width of the ion-cyclotron absorption is typically smaller than in smaller machines and the modification of the RF field polarization caused by changes in the minority fraction tends to have a weaker impact on the wave absorptivity. 

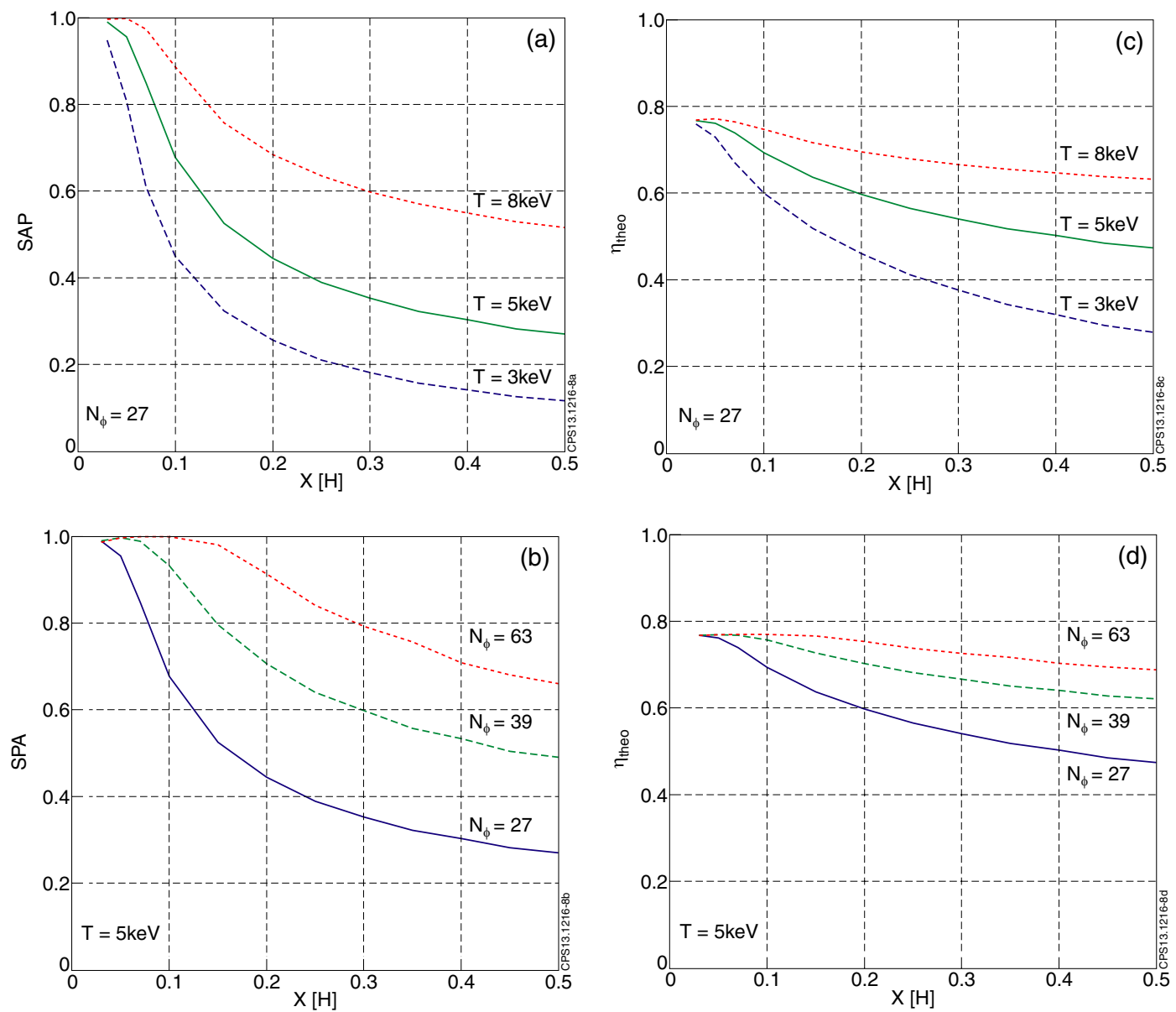

Figure 9. $(a),(b)$ SPA and $(c),(d)$ theoretical heating efficiencies as a function of the $\mathrm{H}$ concentration for different plasma temperatures and different antenna excitations for the fundamental $\mathrm{H}^{-} \mathrm{He}$ ICRF scheme in ITER with $B_{0}=2.7 \mathrm{~T}$ and $42 \mathrm{MHz}$. The heating efficiencies were estimated from the SPA curves considering a multi-pass model with $23 \%$ wave losses per transit in the plasma.

\section{Summary and conclusions}

Recent JET-ILW experiments studied the impact of the minority $\mathrm{H}$ concentration on the fundamental $(\mathrm{H})-\mathrm{D}$ ICRF heating performance in conditions similar to those expected in ITER's initial ${ }^{4} \mathrm{He}$ L-mode operation phase. Since JET is now equipped with a full Be/W ITER-like wall and because the RF settings and the plasma parameters (magnetic field, kinetic profiles) could be closely matched to those expected in ITER, these experiments were a suitable test bed for studying the potential of this heating scenario in ITER. The experiments have shown that, in general, the RF heating efficiency of the $(\mathrm{H}) \mathrm{D}$ ICRF scenario at $B_{0}=2.7 \mathrm{~T} / f=42 \mathrm{MHz}$ in JET-ILW remains comparable to the one measured with the $\mathrm{C}$-wall, despite the larger (off-axis) bulk plasma radiation observed in ICRF heated discharges due to the increased levels of $\mathrm{W}$ (and $\mathrm{Ni}$ ) in the plasma, leading to a slightly lower energy content achieved for the same RF power applied. The heating efficiency degrades from $0.7-0.8$ at low and medium $\mathrm{H}$ concentrations to about 0.4 when $X[\mathrm{H}]=n_{\mathrm{H}} / n_{\mathrm{e}}=30 \%$ is reached, but the decay only starts to be significant at moderate $X[\mathrm{H}] \sim 15 \%$ values. The plasma energy reached per MW of RF power at constant density follows this trend closely and is lower for larger $X[\mathrm{H}]$. The central electron temperatures show a linear decay with $X[\mathrm{H}]$ from very low $\mathrm{H}$ concentrations since on top of their dependence on the heating efficiency, they are also influenced by the change in the fast ion dynamics with $X[\mathrm{H}]$ (different slowing-down). NPA results confirm the sharp drop of the fast ion tail energy when increasing the $\mathrm{H}$ concentration: The tail energies decrease from $350 \mathrm{keV}$ at low $X[\mathrm{H}]$ to below $100 \mathrm{keV}$ at $X[\mathrm{H}]=20 \%$. Interestingly, the high- $Z$ impurity levels and thus the bulk plasma radiation exhibits a minimum at moderate $\mathrm{H}$ concentrations, but due to changes in the plasma edge properties also observed in the experiments the exact cause of this effect remains unclear.

Encouraged by the very good agreement between the JET results and the numerical modelling performed with RF wave and Fokker-Planck codes, estimates for the heating performance of the (H)- ${ }^{4} \mathrm{He}$ ICRF heating scheme for ITER's half-field parameters were done. The simulations indicate that a good performance of this RF heating scenario can be expected in ITER up to high levels of hydrogen, provided the plasma is kept warm enough (pre-heated by other auxiliary heating scheme) or if high $k_{\|}$spectra $(0 \pi \pi 0$ or $0 \pi 0 \pi$ phasing) are used. If assuming the same wave losses per transit as obtained in the JET experiments and the reference ITER temperature of $T_{0}=5 \mathrm{keV}$, the expected heating efficiencies never drop below $\eta_{\text {theo }} \sim 0.5$ within $X[\mathrm{H}]=3-50 \%$.

The experiments have also shown that the fast-particle population is strongly reduced when the $\mathrm{H}$ concentration is 
increased. For ITER, where due to its large volume the central RF power density values remain modest even when $10 \mathrm{MW}$ of ICRF power is applied, the simulations suggest that this drop may be even more pronounced. This is likely to have consequences on the central electron temperatures achieved with auxiliary ICRF heating at large $\mathrm{H}$ fractions and therefore, if high- $Z$ impurity control is already needed at this stage of ITER's operation, ensuring sufficient ICRF power being available (on top of the central ECRF heating foreseen) may become vital from early on [34].

Finally, it is important to mention that the $20-25 \%$ edge power losses estimated in JET are not necessarily directly extrapolatable to ITER since the ICRF antenna design as well as the SOL characteristics in the two machines is different. However, since these losses are present even when the singlepass wave absorption is unity it is crucial that further progress on understanding the edge RF processes (antenna-plasma coupling, RF sheath rectification effects and parasitic power dissipation) is made for minimizing this deleterious effect in future machines.

\section{Acknowledgments}

The support from the JET ICRH system group is warmly acknowledged. This work was carried out within the framework of the European Fusion Development Agreement. The views and opinions expressed herein do not necessarily reflect those of the European Commission.

\section{References}

[1] Polevoi A.R. et al 2008 Proc. 22nd Int. Conf. on Fusion Energy 2008 (Geneva, Switzerland, 2008) (Vienna: IAEA) CD-ROM file IT/P6-6 http://www-naweb.iaea.org/ napc/physics/FEC/FEC2008/papers/it_p6-6.pdf

[2] Lerche E. et al 2011 Plasma Phys. Control. Fusion 53124019

[3] Lerche E. et al 2011 19th Topical RF Conf. (Newport, RI, 2011) AIP Conf. Proc. 1406245
[4] Budny R. et al 2012 Nucl. Fusion 52023023

[5] Bilato R. et al 2014 20th Topical RF Conf. (Sorrento, Italy, 2013) AIP Conf. Proc. 1580291

[6] Lerche E. et al 2012 Plasma Phys. Control. Fusion 54074008

[7] Koch R. et al 2005 Fusion Sci. Technol. 4797

[8] Eriksson L.-G. et al 2001 Nucl. Fusion 4191

[9] Takase Y. et al 1996 Plasma Phys. Control. Fusion 382215

[10] Pinsker R. et al 1992 9th Topical RF Conf. (Charleston, SC, 1991) AIP Conf. Proc. 244105

[11] Start D. et al 1989 8th Topical RF Conf. (Irvine, CA, 1989) AIP Conf. Proc. 190205

[12] Mantsinen M. et al 1999 Plasma Phys. Control. Fusion 41843

[13] Bobkov V. et al 2010 Nucl. Fusion $\mathbf{5 0} 035004$

[14] Matthews G. et al 2011 Phys. Scr. 2011014001

[15] Bobkov V. et al 2013 J. Nucl. Mater. 438 S160-5

[16] Jacquet P. et al 2013 J. Nucl. Mater. 438 S379-83

[17] Jacquet P. et al 2014 Phys. Plasmas at press

[18] Mayoral M.-L. et al 2014 Nucl. Fusion 54033002

[19] Van Eester D. et al 2014 20th Topical RF Conf. (Sorrento, Italy, 2013) AIP Conf. Proc. 1580223

[20] Lamalle P. 1994 LPP-ERM/KMS Lab. Report 101, Brussels

[21] Lerche E. et al 2009 Plasma Phys. Control. Fusion 51044006

[22] Van Eester D. et al 1998 Plasma Phys. Control. Fusion 401949

[23] Van Eester D. et al 2011 Plasma Phys. Control. Fusion 53092001

[24] Loarer T. et al 2013 J. Nucl. Mater. 438 S108-13

[25] Solano E. et al 2013 Proc. 40th EPS Conf. on Plasma Physics (Helsinki, Finland, 2013) vol 37D (ECA) P4.111 http://ocs.ciemat.es/EPS2013PAP/html/contrib.html

[26] Czarnecka A. et al 2014 20th Topical RF Conf. (Sorrento, Italy, 2013) AIP Conf. Proc. 1580227

[27] Czarnecka A. et al 2012 Plasma Phys. Control. Fusion 54074013

[28] Lerche E. et al 2008 Plasma Phys. Control. Fusion $\mathbf{5 0} 035003$

[29] Korotkov A. et al 1997 Nucl. Fusion 3735

[30] Graves J. et al 2010 Nucl. Fusion $\mathbf{5 0} 052002$

[31] Van Eester D. et al 2013 Plasma Phys. Control. Fusion $\mathbf{5 5} 055008$

[32] Stix T. 1975 Nucl. Fusion 15737

[33] Karney C. 1986 Comput. Phys. Rep. 4 183-244

[34] Messiaen A. et al 2010 Nucl. Fusion 50025026

[35] Valisa M. et al 2011 Nucl. Fusion 51033002 